\title{
COMPLEX NILPOTENT LEIBNIZ SUPERALGEBRAS WITH NILINDEX EQUAL TO DIMENSION
}

\author{
L. M. Camacho' ${ }^{1}$, J. R. Gómez ${ }^{1}$, B. A. Omirov², \\ and A. Kh. Khudoyberdiyev ${ }^{2}$ \\ ${ }^{1}$ Dpto. Matemática Aplicada I, Universidad de Sevilla, Sevilla, Spain ${ }^{2}$ Institute \\ of Mathematics and Information Technologies of Academy of Uzbekistan, \\ Tashkent, Uzbekistan
}

\begin{abstract}
We present the description up to isomorphism of Leibniz superalgebras with the characteristic sequence equal to $\left(n \mid m_{1}, \ldots, m_{k}\right)$ and nilindex $n+m$, where $m=m_{1}+$ $\cdots+m_{k}$, and $n$ and $m(m \neq 0)$ are the dimensions of the even and odd parts, respectively.
\end{abstract}

Key Words: Characteristic sequence; Leibniz superalgebras; Lie superalgebras; Nilindex.

\section{INTRODUCTION}

We know that Lie algebras are generalized in several ways. We may change the Jacobi identity to the Malcev identity (in this case, we get Malcev algebras [16]). We may also omit the skew-symmetric identity, and transforming the Jacobi identity we get Leibniz algebra $[6,15]$. If we consider the graded skew-symmetric and Jacobi identities, we can derive Lie superalgebras. The concept of Leibniz superalgebra and its cohomology was first introduced by Dzhumadil'daev in [7]. In the present article, that we study nilpotent Leibniz superalgebras. Recall that Leibniz superalgebras are constructed by the graded Leibniz identity and therefore they include Leibniz algebras and Lie superalgebras.

Since nilpotent Leibniz superalgebras are defined by the descending central sequence, it is natural to consider the descending filtration which consists of degrees of the superalgebra. When the length is equal to two, i.e., the numbers of the members of filtration are equal to two, we get the trivial superalgebra. For an $(n+m)$-dimensional Leibniz superalgebra the maximum length of the filtration is equal to $n+m+1$ with $n$ and $m$ are the dimensions of the even and odd parts of the superalgebra, respectively. Such Leibniz superalgebras have a simple structure (see [1]), and they are single-generated. It should be noted that Lie superalgebras have more than one generator. Note that the structure of superalgebras is simpler 
when the length of the filtration is bigger than when it is smaller. Indeed, the problem of the classification of complex 5-dimensional associative algebras with the length of the filtration is equal to three is still open. It should be noted that many important properties of Leibniz algebras depend on the operator of right multiplications, which is a derivation. In [11] the authors show that the adjoint operators of a general algebra are derivations if and only if the algebra is a Leibniz algebras.

In other words, the nilindex is nothing else but the length of the descending filtration. Therefore, it is natural to consider Leibniz superalgebras of nilindex $n+m$.

The set of all nilpotent $(n+m)$-dimensional Leibniz superalgebras forms a variety [1]. From algebraic geometry it is known that the variety is a union of finite numbers of irreducible components [13]. The representatives of the irreducible components are the so-called rigid superalgebras, from which we can construct its irreducible component $[2,10]$. In fact, the first candidates to rigid superalgebras are those superalgebras which have the biggest nilindex. Lie superalgebras with the length of the filtration equal to $n+m$ lead to the existence of only one superalgebra [9]. The study of non Lie Leibniz superalgebras involves serious difficulties because we do not have the graded skew-symmetric identity. That is why we need to add some additional restrictions. Nilpotent Leibniz superalgebras are studied in papers $[4,5,8]$. The even part of such superalgebras have maximal length of the filtration and in each dimension there exists a unique non Lie Leibniz algebra [3]. In order to study Leibniz superalgebras of nilindex $n+m$, we start by those with characteristic sequence $\left(n \mid m_{1}, m_{2}, \ldots, m_{k}\right)$.

Taking into account the results of papers [5, 8], where some cases of nilpotent Leibniz superalgebras were described, we investigate the remaining cases. Thus, the present article completes the classification of Leibniz superalgebras with characteristic sequence $\left(n \mid m_{1}, \ldots, m_{k}\right)$ and nilindex $n+m$.

Throughout this article, we consider linear spaces and algebras over the field of complex numbers.

\section{PRELIMINARIES}

Lie and Leibniz superalgebras are defined as follows.

Definition 2.1 ([14]). A $\mathbb{Z}_{2}$-graded vector space $\mathscr{G}=\mathscr{G}_{0} \oplus \mathscr{G}_{1}$ is called a Lie superalgebra if it is equipped with a product $[-,-]$ which satisfies the following conditions:

1. $\left[\mathscr{G}_{\alpha}, \mathscr{G}_{\beta}\right] \subseteq \mathscr{G}_{\alpha+\beta}$;

2. $[x, y]=-(-1)^{\alpha \beta}[y, x]$ - graded skew-symmetric identity;

3. $(-1)^{\alpha \gamma}[x,[y, z]]+(-1)^{\alpha \beta}[y,[z, x]]+(-1)^{\beta \gamma}[z,[x, y]]=0-$ Jacobi superidentity for any $x \in \mathscr{G}_{\alpha}, y \in \mathscr{G}_{\beta}, z \in \mathscr{G}_{\gamma}$, and $\alpha, \beta, \gamma \in \mathbb{Z}_{2}$.

Definition 2.2 ([1]). A $\mathbb{Z}_{2}$-graded vector space $\mathscr{L}=\mathscr{L}_{0} \oplus \mathscr{L}_{1}$ is called a Leibniz superalgebra if it is equipped with a product $[-,-]$ which satisfies the following conditions:

1. $\left[\mathscr{L}_{\alpha}, \mathscr{L}_{\beta}\right] \subseteq \mathscr{L}_{\alpha+\beta} ;$ 
2. $[x,[y, z]]=[[x, y], z]-(-1)^{\alpha \beta}[[x, z], y]-$ Leibniz superidentity, for all $x \in \mathscr{L}, y \in$ $\mathscr{L}_{\alpha}, z \in \mathscr{L}_{\beta}$, and $\alpha, \beta \in \mathbb{Z}_{2}$.

The vector spaces $\mathscr{L}_{0}$ and $\mathscr{L}_{1}$ are said to be the even and odd parts of the superalgebra $\mathscr{L}$, respectively.

Note that if in $\mathscr{L}$ the graded skew-symmetric identity holds, then the Leibniz superidentity and the Jacobi superidentity coincide. Thus, Leibniz superalgebras are a generalization of Lie superalgebras.

For examples of Leibniz superalgebras see [1].

We denote by Leib ${ }^{n, m}$ the set of Leibniz superalgebras with the dimensions of the even part and the odd part equal to $n$ and $m$, respectively.

Definition 2.3. Let $\mathscr{L}$ and $\mathscr{L}^{\prime}$ be Leibniz superalgebras. A linear map $f: \mathscr{L} \rightarrow \mathscr{L}^{\prime}$ is called a homomorphism of Leibniz superalgebras if:

1. $f\left(\mathscr{L}_{0}\right) \subseteq \mathscr{L}_{0}^{\prime}$ and $f\left(\mathscr{L}_{1}\right) \subseteq \mathscr{L}_{1}^{\prime}$;

2. $f([x, y])=[f(x), f(y)]$ for all $x, y \in \mathscr{L}$.

Moreover, if $f$ is a bijection, then it is called an isomorphism of Leibniz superalgebras $\mathscr{L}$ and $\mathscr{L}^{\prime}$.

For a given Leibniz superalgebra $\mathscr{L}$, we define the descending central sequence as follows:

$$
\mathscr{L}^{1}=\mathscr{L}, \quad \mathscr{L}^{k+1}=\left[\mathscr{L}^{k}, \mathscr{L}^{1}\right], \quad k \geq 1 .
$$

Definition 2.4. A Leibniz superalgebra $\mathscr{L}$ is called nilpotent, if there exists $t \in \mathbb{N}$ such that $\mathscr{L}^{t}=0$. The smaller number $t$ with this property is called the nilindex of the superalgebra $\mathscr{L}$.

Note that the nilindex of the nilpotent superalgebra of $L e i b^{n, m}$ is less or equal to $n+m+1$.

Definition 2.5. The sets

$$
\mathscr{R}(\mathscr{L})=\{z \in \mathscr{L} \mid[\mathscr{L}, z]=0\} \quad \text { and } \quad \mathscr{L}(\mathscr{L})=\{z \in \mathscr{L} \mid[\mathscr{L}, z]=[z, \mathscr{L}]=0\}
$$

are called the right annihilator and the center of a superalgebra $\mathscr{L}$, respectively.

It is easy to check that $\mathscr{R}(\mathscr{L})$ is an ideal of the superalgebra $\mathscr{L}$ and the elements of the form $[a, b]+(-1)^{\alpha \beta}[b, a],\left(a \in \mathscr{L}_{\alpha}, b \in \mathscr{L}_{\beta}\right)$ belong to $\mathscr{R}(\mathscr{L})$.

The following theorem describes nilpotent Leibniz superalgebras with maximal nilindex.

Theorem 2.6 ([1]). Let $\mathscr{L}$ be a nilpotent Leibniz superalgebra of the variety Leib $^{n, m}$ with nilindex equal to $n+m+1$. Then $\mathscr{L}$ is isomorphic to one of the following non- 
isomorphic superalgebras:

$$
\left[e_{i}, e_{1}\right]=e_{i+1}, \quad 1 \leq i \leq n-1 ; \quad \begin{cases}{\left[e_{i}, e_{1}\right]=e_{i+1},} & 1 \leq i \leq n+m-1 \\ {\left[e_{i}, e_{2}\right]=2 e_{i+2},} & 1 \leq i \leq n+m-2\end{cases}
$$

(where the products equal to zero are omitted).

It should be noted that for the second superalgebra we have $m=n$ when $n+m$ is even and $m=n+1$ if $n+m$ is odd. Moreover, the Leibniz superalgebra (algebra) has the maximal nilindex if and only if it has only one generator.

Let $\mathscr{L}=\mathscr{L}_{0} \oplus \mathscr{L}_{1}$ be a complex nilpotent Leibniz superalgebra. For an arbitrary element $x \in L_{0}$, the operator of right multiplication $R_{x}: \mathscr{L} \rightarrow \mathscr{L}$ (defined by $\left.R_{x}(y)=[y, x]\right)$ is a nilpotent endomorphism of the space $L_{i}$, where $i \in\{0,1\}$. Taking into account the property of complex field, we can consider the Jordan form for $R_{x}$. Denote by $C_{i}(x)(i \in\{0,1\})$ the descending sequence of the Jordan blocks dimensions of $R_{x}$. Consider the lexicographical order on the set $C_{i}\left(\mathscr{L}_{0}\right)=\left\{C_{i}(x) \mid x \in\right.$ $\left.L_{0}\right\}, i \in\{0,1\}$. Let us define the notion of characteristic sequence for nilpotent Leibniz superalgebras, which is first introduced for the nilpotent Lie algebras in [12].

Definition 2.7. A sequence

$$
C(\mathscr{L})=\left(\max _{\left.x \in \mathscr{L}_{0} \backslash \backslash \mathscr{I}_{0}, \mathscr{L}_{0}\right]} C_{0}(x) \mid \max _{\tilde{x} \in \mathscr{I}_{0} \backslash\left[\mathscr{L}_{0}, \mathscr{L}_{0}\right]} C_{1}(\tilde{x})\right)
$$

is said to be the characteristic sequence of the Leibniz superalgebra $\mathscr{L}$.

For Leibniz superalgebras, we introduce the analogue of the zero-filiform Leibniz algebras.

Definition 2.8. A Leibniz superalgebra $\mathscr{L} \in L e i b^{n, m}$ is called zero-filiform if $C(\mathscr{L})=(n \mid m)$.

Denote by $\mathscr{Z F}^{n, m}$ the set of all zero-filiform Leibniz superalgebras from $L e i b^{n, m}$.

From [3] it can be concluded that the even part of zero-filiform Leibniz superalgebras is a zero-filiform Leibniz algebra; therefore, zero-filiform superalgebras are not Lie superalgebras.

Further, we need the following result on the existence of an adapted basis for zero-filiform Leibniz superalgebras. 
Theorem 2.9 ([8]). In an arbitrary superalgebra from $\mathscr{Z F F}^{n, m}$ there exists a basis $\left\{x_{1}, x_{2}, \ldots, x_{n}, y_{1}, y_{2}, \ldots, y_{m}\right\}$, which satisfies the following conditions:

$$
\begin{cases}{\left[x_{i}, x_{1}\right]=x_{i+1},} & 1 \leq i \leq n-1, \\ {\left[x_{n}, x_{1}\right]=0,} & \\ {\left[x_{i}, x_{k}\right]=0,} & 1 \leq i \leq n, \quad 2 \leq k \leq n \\ {\left[y_{j}, x_{1}\right]=y_{j+1},} & 1 \leq j \leq m-1, \\ {\left[y_{m}, x_{1}\right]=0,} & \\ {\left[y_{j}, x_{k}\right]=0,} & 1 \leq j \leq m, \quad 2 \leq k \leq n\end{cases}
$$

From Theorem 2.9, it is not difficult to see that, for the zero-filiform Leibniz algebra,

$$
C(\mathscr{L})=\left(C_{0}\left(x_{1}\right) \mid C_{1}\left(x_{1}\right)\right)
$$

But in general, in the definition characteristic sequence $C(\mathscr{L})$ the elements $x$ and $\tilde{x}$ are distinct.

\section{ZERO-FILIFORM LEIBNIZ SUPERALGEBRAS OF LEIB ${ }^{n, m}$ WITH NILINDEX $\boldsymbol{n}+\boldsymbol{m}$}

This section is devoted to the description of zero-filiform Leibniz superalgebras of $L e i b^{n, m}$ with nilindex equal to $n+m$.

Let $\mathscr{L} \in \mathscr{E}^{n, m}$ with nilindex equal to $n+m$. Note that the Leibniz superalgebras in Theorem 2.6 are zero-filiform, which have nilindex equal to $n+$ $m+1$, and therefore, they are one-generated superalgebras. Thus, the Leibniz superalgebras with nilindex $n+m$, have at least two generators. If the number of generators is more than two, then the superalgebra has nilindex less than $n+m$. Hence, we have that $\mathscr{L}$ has exactly two generators. Moreover, from Theorem 2.9, it follows that one generator lies in $\mathscr{L}_{0}$, and the second generator lies in $\mathscr{L}_{1}$. Without loss of generality, it can be assumed that in the adapted basis the generators are $x_{1}$ and $y_{1}$. Then we have $\mathscr{L}^{2}=\left\{x_{2}, x_{3}, \ldots, x_{n}, y_{2}, y_{3}, \ldots, y_{m}\right\}$.

In the adapted basis of $\mathscr{L}$, we introduce the notations:

$$
\left[x_{i}, y_{1}\right]=\sum_{j=2}^{m} \alpha_{i, j} y_{j}, \quad 1 \leq i \leq n, \quad\left[y_{i}, y_{1}\right]=\sum_{j=2}^{n} \beta_{i, j} x_{j}, \quad 1 \leq i \leq m .
$$

Thus, the following lemma holds.

\section{Lemma 3.1.}

$$
\left[y_{i}, y_{j}\right]=\sum_{s=0}^{\min \{i+j-1, m\}-i}(-1)^{s} C_{j-1}^{s} \sum_{t=2}^{n-j+s+1} \beta_{i+s, t} x_{t+j-s-1},
$$

where $1 \leq i, j \leq m$ and $C_{j-1}^{s}=\frac{(j-1) !}{s !(j-1-s) !}$ are the binomial coefficients.

Proof. The proof is deduced by $j$ induction for any value of $i$. 
Since the set $\mathscr{E}^{n, 2}$ was already described in [8], we consider the set $\mathscr{Z F}^{n, m}$ with $m \geq 3$.

Case $\mathscr{E F}^{2, m}(m \geq 3)$.

Theorem 3.2. Let $\mathscr{L} \in \mathscr{L F}^{2, m}(m \geq 3)$ with nilindex $m+2$. Then $m$ is odd and $\mathscr{L}$ is isomorphic to the following superalgebra:

$$
\begin{cases}{\left[x_{1}, x_{1}\right]=x_{2},} & 1 \leq i \leq m-1 \\ {\left[y_{i}, x_{1}\right]=y_{i+1},} & 1 \leq i \leq m-1 \\ {\left[x_{1}, y_{i}\right]=-y_{i+1},} & 1 \leq i \leq m-1 \\ {\left[y_{i}, y_{m+1-i}\right]=(-1)^{j+1} x_{2},} & \end{cases}
$$

Proof. From (1), we easily obtain

$$
\begin{aligned}
& {\left[y_{i}, y_{j}\right]=(-1)^{j-1} \beta_{i+j-1,2} x_{2}, \quad 2 \leq i+j \leq m+1,} \\
& {\left[y_{i}, y_{j}\right]=0, \quad m+2 \leq i+j \leq 2 m .}
\end{aligned}
$$

It should be noted that $\beta_{m, 2} \neq 0$. Indeed, if $\beta_{m, 2}=0$, then $\mathscr{L}^{m-1}=$ $\left\{x_{2}, y_{m-1}, y_{m}\right\}, \mathscr{L}^{m}=\left\{x_{2}, y_{m}\right\}$, and $\mathscr{L}^{m+1}=\left\{y_{m}\right\}$, which implies that $\left[y_{m-1}, y_{1}\right]=a x_{2}$ and $\left[x_{2}, y_{1}\right]=b y_{m}$, where $a b \neq 0$.

The chain of the equalities

$$
a b y_{m}=\left[a x_{2}, y_{1}\right]=\left[\left[y_{m-1}, y_{1}\right], y_{1}\right]=\frac{1}{2}\left[y_{m-1},\left[y_{1}, y_{1}\right]\right]=0
$$

contradicts the property $a b \neq 0$. Therefore, $\beta_{m, 2} \neq 0$.

The simple analysis of the products leads to $x_{2} \in \mathscr{L}(\mathscr{L})$ (since $x_{2} \in \mathscr{L}^{m+1} \subseteq$ $\mathscr{Z}(\mathscr{L})$ ).

Using the Leibniz superidentity, we have

$$
\left[x_{1}, y_{i}\right]=\alpha_{1,2} y_{i+1}+\cdots+\alpha_{1, m-i+1} y_{m}, \quad 1 \leq i \leq m-1 .
$$

The expression $\left[y_{1}, x_{1}\right]+\left[x_{1}, y_{1}\right]$ lies in $\mathscr{R}(\mathscr{L})$. Hence

$$
\left(1+\alpha_{1,2}\right) y_{2}+\alpha_{1,3} y_{3}+\cdots+\alpha_{1, m} y_{m}
$$

belongs to $\mathscr{R}(\mathscr{L})$, as well.

If either $\alpha_{1,2} \neq-1$ or there exists $(3 \leq i \leq m)$ such that $\alpha_{1, i} \neq 0$, we deduce $y_{m} \in \mathscr{R}(\mathscr{L})$ multiplying (3) by $x_{1}$ conveniently from the right side. However, by (2) we have $\left[y_{1}, y_{m}\right]=(-1)^{m-1} \beta_{m, 2} x_{2}$, which implies that $\beta_{m, 2}=0$, and we get a contradiction with condition $\beta_{m, 2} \neq 0$.

If $\alpha_{1,2}=-1$ and $\alpha_{1, i}=0 \quad(3 \leq i \leq m)$, then by applying the Leibniz superidentity for the basis elements $\left\{x_{1}, y_{i}, y_{i}\right\}$, we obtain $\beta_{2 i, 2}=0$ for $1 \leq i \leq\left[\frac{m}{2}\right]$.

Note that if $m$ is even, then we obtain $\beta_{m, 2}=0$, which is a contradiction. Therefore, $m$ is odd. 
Let us introduce some new notations:

$$
\gamma_{s}=\beta_{2 s-1,2}, \quad 1 \leq s \leq \frac{m+1}{2} .
$$

Then we obtain the family $L\left(\gamma_{1}, \gamma_{2}, \ldots, \gamma_{\frac{m+1}{2}}\right)$ :

$$
\begin{cases}{\left[x_{1}, x_{1}\right]=x_{2},} & \\ {\left[y_{i}, x_{1}\right]=y_{i+1},} & 1 \leq i \leq m-1, \\ {\left[x_{1}, y_{i}\right]=-y_{i+1},} & 1 \leq i \leq m-1, \\ {\left[y_{i}, y_{j}\right]=(-1)^{j-1} \gamma_{\frac{i+j}{2}} x_{2},} & i+j \text { is even, } 2 \leq i+j \leq m+1, m \text { is odd }\end{cases}
$$

Make the following general transformation of the generators:

$$
x_{1}^{\prime}=b_{1} x_{1}, \quad y_{1}^{\prime}=\sum_{s=1}^{\frac{m+1}{2}} a_{2 s-1} y_{2 s-1} .
$$

Then $x_{2}^{\prime}=b_{1}^{2} x_{2}$ and

$$
\begin{aligned}
y_{2 i-1}^{\prime}=b_{1}^{2(i-1)} \sum_{s=1}^{\frac{m-2(i-1)+1}{2}} a_{2 s-1} y_{2 s+2 i-3}, & 1 \leq i \leq \frac{m+1}{2}, \\
y_{2 i}^{\prime}=b_{1}^{2 i-1} \frac{\frac{m-2(i-1)-1}{2}}{\sum_{s=1}^{2}} a_{2 s-1} y_{2 s+2 i-2}, & 1 \leq i \leq \frac{m-1}{2} .
\end{aligned}
$$

We obtain $\left[y_{m}^{\prime}, y_{1}^{\prime}\right]=x_{2}^{\prime}$ and $\left[y_{i}^{\prime}, y_{1}^{\prime}\right]=0$ for $1 \leq i \leq m-1$, choosing the parameters $a_{i}$ as follows:

$$
\begin{aligned}
a_{1}= & \sqrt{\frac{1}{b_{1}^{m-3} \gamma_{\frac{m+1}{2}}}}, \quad a_{3}=-\frac{a_{1} \gamma_{\frac{m-1}{2}}}{2 \gamma_{\frac{m+1}{2}}}, \\
a_{i}= & -\frac{a_{1}^{2} \gamma_{\frac{m-i+2}{2}}+2 a_{1} a_{3} \gamma_{\frac{m-i+4}{2}}+\cdots+\left(2 a_{1} a_{i-2}+\cdots+2 a_{\frac{i-3}{2}} a_{\frac{i+1}{2}}\right) \gamma_{\frac{m-1}{2}}}{2 a_{1} \gamma_{\frac{m+1}{2}}} \\
& -\frac{2 a_{3} a_{i-2}+\cdots+2 a_{\frac{i-3}{2}} a_{\frac{i+5}{2}}+a_{\frac{i+1}{2}}^{2}}{2 a_{1}}, \quad \text { if } \frac{i+1}{2} \text { odd and } 4 \leq i \leq m, \\
a_{i}= & -\frac{a_{1}^{2} \gamma_{\frac{m-i+2}{2}}+2 a_{1} a_{3} \gamma_{\frac{m-i+4}{2}}+\cdots+\left(2 a_{1} a_{i-2}+\cdots+2 a_{\frac{i-5}{2}} a_{\frac{i+3}{2}}+a_{\frac{i-1}{2}}^{2}\right) \gamma_{\frac{m-1}{2}}}{2 a_{1} \gamma_{\frac{m+1}{2}}} \\
& -\frac{2 a_{3} a_{i-2}+\cdots+2 a_{\frac{i-1}{2}} a_{\frac{i+3}{2}}}{2 a_{1}}, \quad \text { if } \frac{i+1}{2} \quad \text { even and } 4 \leq i \leq m .
\end{aligned}
$$

Then applying the Leibniz superidentity, we get the remaining brackets

$$
\begin{aligned}
& {\left[y_{i}^{\prime}, y_{j}^{\prime}\right]=0, \quad 1 \leq i, j \leq m, \quad i+j \neq m+1,} \\
& {\left[y_{i}^{\prime}, y_{j}^{\prime}\right]=(-1)^{j-1} x_{2}^{\prime}, \quad 1 \leq i, j \leq m, \quad i+j=m+1 .}
\end{aligned}
$$


Thus, we obtain the superalgebra of the theorem.

Case $\mathscr{Z F}^{n, m}(n \geq 3, m \geq 3)$.

Lemma 3.3. Any Leibniz superalgebra from $\mathscr{E}^{n, m}(n \geq 3, m \geq 3)$ has nilindex less than $n+m$.

Proof. Let $\mathscr{L} \in \mathscr{Z F}^{n, m}$ ( $n \geq 3, m \geq 3$ ) be. Let us assume that $\mathscr{L}$ has nilindex $n+m$. Then we have the following in the adapted basis

$$
\begin{aligned}
\mathscr{L} & =\left\{x_{1}, x_{2}, \ldots, x_{n}, y_{1}, y_{2}, \ldots, y_{m}\right\}, \\
\mathscr{L}^{2} & =\left\{x_{2}, \ldots, x_{n}, y_{2}, \ldots, y_{m}\right\}, \\
\mathscr{L}^{3} & \supset\left\{x_{3}, \ldots, x_{n}, y_{3}, \ldots, y_{m}\right\} .
\end{aligned}
$$

Let us suppose that $\mathscr{L}^{3}=\left\{x_{3}, \ldots, x_{n}, y_{2}, \ldots, y_{m}\right\}$, i.e., $x_{2} \notin \mathscr{L}^{3}$ and $y_{2} \in \mathscr{L}^{3}$. Then there exists $i_{0}\left(2 \leq i_{0} \leq n\right)$ such that $\left[x_{i_{0}}, y_{1}\right]=\alpha_{i_{0}, 2} y_{2}+\cdots+\alpha_{i_{0}, m} y_{m}$ with $\alpha_{i_{0}, 2} \neq 0$. Since $x_{i} \in \mathscr{R}(\mathscr{L})$ for $2 \leq i \leq n$ and $\mathscr{R}(\mathscr{L})$ is an ideal, then $\alpha_{i_{0}, 2} y_{2}+\cdots+$ $\alpha_{i-0, m} y_{m} \in \mathscr{R}(\mathscr{L})$.

Multiplying the product $\left[x_{i_{0}}, y_{1}\right]$ on the right side by $x_{1}(m-1)$-times, we easily obtain $y_{2}, y_{3}, \ldots, y_{m} \in \mathscr{R}(\mathscr{L})$, that is $\mathscr{L}^{2}=\mathscr{R}(\mathscr{L})$.

By induction one can prove the following

$$
\left[x_{i}, y_{1}\right]= \begin{cases}\sum_{j=2}^{m+1-i} \alpha_{1, j} y_{j+i-1}, & \text { if } \quad i+1 \leq m, \\ 0, & \text { if } i+1>m .\end{cases}
$$

Since $\mathscr{L}^{2}=\mathscr{R}(\mathscr{L})$, then $y_{2}$ can appear only in the products $\left[x_{i}, y_{1}\right]$ for $2 \leq$ $i \leq n$ or $\left[y_{j}, x_{1}\right]$ for $2 \leq j \leq m-1$. However, from (4) we conclude that $y_{2}$ does not lie in $\mathscr{L}^{3}$ in the first case and in the second case the element $y_{2}$ cannot be obtained, i.e. in both cases we have a contradiction with the assumption $\mathscr{L}^{3}=$ $\left\{x_{3}, \ldots, x_{n}, y_{2}, \ldots, y_{m}\right\}$. $\mathscr{L}^{s} \backslash \mathscr{L}^{s+1}$.

Thus, $\mathscr{L}^{3}=\left\{x_{2}, \ldots, x_{n}, y_{3}, \ldots, y_{m}\right\}$. Let $s$ be a natural number such that $x_{2} \in$

Suppose $s \leq m$. Then we have

$$
\begin{aligned}
\mathscr{L}^{i} & =\left\{x_{2}, \ldots, x_{n}, y_{i}, \ldots, y_{m}\right\}, \quad 2 \leq i \leq s, \\
\mathscr{L}^{s+1} & =\left\{x_{3}, \ldots, x_{n}, y_{s}, \ldots, y_{m}\right\}
\end{aligned}
$$

and in the equality $\left[y_{s-1}, y_{1}\right]=\sum_{j=2}^{n} \beta_{s-1, j} x_{j}$ the coefficient $\beta_{s-1,2}$ is not zero.

From Lemma 3.1, we have

$$
\left[y_{1}, y_{s}\right]=\sum_{i=0}^{s-1}(-1)^{i} C_{s-1}^{i} \sum_{t=2}^{n-s+i+1} \beta_{1+i, t} x_{t+s-i-1},
$$

in which the coefficient $\beta_{s-1,2}$ occurs. Taking into account the equality $\left[y_{s}, y_{1}\right]=$ $\sum_{j=2}^{n} \beta_{s, j} x_{j}$, we conclude that $x_{3} \in\left\{\left[y_{1}, y_{s}\right],\left[y_{s}, y_{1}\right], x_{4}, \ldots, x_{n}\right\}$. Therefore, $\mathscr{L}^{s+2}=$ $\left\{x_{3}, \ldots, x_{n}, y_{s+1}, \ldots, y_{m}\right\}$, i.e., $y_{s} \in \mathscr{L}^{s+1} \backslash \mathscr{L}^{s+2}$ and $\alpha_{2, s} \neq 0$. 
Consider the equalities

$$
\begin{aligned}
{\left[y_{s-1},\left[y_{1}, y_{1}\right]\right] } & =2\left[\left[y_{s-1}, y_{1}\right], y_{1}\right] \\
& =2 \beta_{s-1,2}\left[x_{2}, y_{1}\right]+\sum_{t=3}^{n} \beta_{s-1, t}\left[x_{t}, y_{1}\right] \\
& \equiv 2 \beta_{s-1,2} \alpha_{2, s} y_{s}\left(\bmod V_{s+1}\right),
\end{aligned}
$$

where $V_{j}=\left\{y_{j}, y_{j+1}, \ldots, y_{m}\right\}$ with $1 \leq j \leq m$.

On the other hand, $\left[y_{s-1},\left[y_{1}, y_{1}\right]\right]=0$, because $\left[y_{1}, y_{1}\right] \in \mathscr{R}(\mathscr{L})$. Hence we have that $\beta_{s-1,2} \alpha_{2, s} y_{s} \equiv 0\left(\bmod V_{s+1}\right)$, which implies $\beta_{s-1,2} \alpha_{2, s}=0$. This contradicts the assumption $s \leq m$.

Let us consider now the case $s=m+1$. Then we have

$$
\begin{aligned}
\mathscr{L} & =\left\{x_{1}, x_{2}, \ldots, x_{n}, y_{1}, y_{2}, \ldots, y_{m}\right\}, \\
\mathscr{L}^{i} & =\left\{x_{2}, x_{3}, \ldots, x_{n}, y_{i}, y_{i+1} \ldots, y_{m}\right\}, \quad 2 \leq i \leq m, \\
\mathscr{L}^{m+i-1} & =\left\{x_{i}, x_{i+1} \ldots, x_{n}\right\}, \quad 2 \leq i \leq n .
\end{aligned}
$$

Since $x_{2} \in \mathscr{L}^{m+1}$ we have $\left[y_{m}, y_{1}\right]=\sum_{i=2}^{n} \beta_{m, i} x_{i}$ with $\beta_{m, 2} \neq 0$.

The sum $\left[y_{1}, x_{1}\right]+\left[x_{1}, y_{1}\right]$ lies in $\mathscr{R}(\mathscr{L})$ since $\left[y_{1}, x_{1}\right]+\left[x_{1}, y_{1}\right]=\left(1+\alpha_{1,2}\right) y_{2}+$ $\alpha_{1,3} y_{3}+\cdots+\alpha_{1, m} y_{m} \in \mathscr{R}(\mathscr{L})$.

If $\left[y_{1}, x_{1}\right]+\left[x_{1}, y_{1}\right]=0$, then using the Leibniz superidentity we have

$\left[x_{1},\left[y_{m}, y_{1}\right]\right]=\left[\left[x_{1}, y_{m}\right], y_{1}\right]+\left[\left[x_{1}, y_{1}\right], y_{m}\right]=-\left[y_{2}, y_{m}\right] \equiv \beta_{m, 2} x_{3}\left(\bmod U_{4}\right)$,

where $U_{i}=\left\{x_{i}, x_{i+1}, \ldots, x_{n}\right\}, 1 \leq i \leq n$.

On the other hand,

$$
\left[x_{1},\left[y_{m}, y_{1}\right]\right]=\sum_{i=2}^{n} \beta_{m, i}\left[x_{1}, x_{i}\right]=0 .
$$

Hence, $\beta_{m, 2}=0$, which is a contradiction.

Thus, $\left[y_{1}, x_{1}\right]+\left[x_{1}, y_{1}\right] \neq 0$. Continuing the same argumentation as in the proof of Theorem 3.2 we obtain $y_{m} \in \mathscr{R}(\mathscr{L})$. Therefore,

$$
\left[y_{1}, y_{m}\right]=\sum_{i=0}^{m-1}(-1)^{i} C_{m-1}^{i} \sum_{t=2}^{n-m+i+1} \beta_{1+i, t} x_{t+m-i-1}=0 .
$$

The minimal value of the expression $t+m-i-1$ is reached when $i=m-1$ and $t=2$. Thus, $\left[y_{1}, y_{m}\right] \equiv(-1)^{m-1} C_{m-1}^{m-1} \beta_{m, 2} x_{2}\left(\bmod U_{3}\right)$ which implies that $\beta_{m, 2}=0$. That is a contradiction with the assumption that the nilindex of $\mathscr{L}$ is equal to $n+m$.

\section{LEIBNIZ SUPERALGEBRAS WITH CHARACTERISTIC SEQUENCE $\left(n \mid m_{1}, m_{2}, \ldots, m_{k}\right)$ AND NILINDEX $n+m$}

Leibniz superalgebras with characteristic sequence $(n \mid m-1,1)$ and with nilindex $n+m$ were examined in [5]. Therefore, in this section we shall consider 
the Leibniz superalgebras $\mathscr{L}$ of nilindex $n+m$ with characteristic sequence $\left(n \mid m_{1}, m_{2}, \ldots, m_{k}\right)$ with the condition $m_{1} \leq m-2$.

From the characteristic sequence, there exits a basis $\left\{x_{1}, x_{2}, \ldots x_{n}, y_{1}, y_{2}, \ldots y_{m}\right\}$ where the operator $R_{\left.x_{1}\right|_{L_{1}}}$ has the following form:

$$
R_{x_{1} \mid \mathscr{s}_{1}}=\left(\begin{array}{cccc}
J_{m_{1}} & 0 & \cdots & 0 \\
0 & J_{m_{2}} & \cdots & 0 \\
\cdots & \cdots & \cdots & \cdots \\
0 & 0 & \cdots & J_{m_{k}}
\end{array}\right)
$$
conditions:

It means that the basis $\left\{x_{1}, x_{2}, \ldots x_{n}, y_{1}, y_{2}, \ldots y_{m}\right\}$ satisfies the following

$$
\begin{aligned}
& {\left[x_{i}, x_{1}\right]=x_{i+1}, \quad 1 \leq i \leq n-1,} \\
& {\left[y_{j}, x_{1}\right]=y_{j+1}, \quad \text { for } j \notin\left\{m_{1}, m_{1}+m_{2}, \ldots, m_{1}+m_{2}+\cdots+m_{k}\right\},} \\
& {\left[y_{j}, x_{1}\right]=0, \quad \text { for } j \in\left\{m_{1}, m_{1}+m_{2}, \ldots, m_{1}+m_{2}+\cdots+m_{k}\right\} .}
\end{aligned}
$$

It is clear that the two generators cannot lie in $\mathscr{L}_{0}$. In fact, in [3] the table of multiplication of the Leibniz algebra $\mathscr{L}_{0}$ is presented, and it has only one generator.

Theorem 4.1. Let $\mathscr{L}$ be a Leibniz superalgebra of nilindex $n+m$ with characteristic sequence $\left(n \mid m_{1}, m_{2}, \ldots, m_{k}\right)$, where $m_{1} \leq m-2$. Then both generators cannot belong to $\mathscr{L}_{1}$ simultaneously.

Proof. Let $\mathscr{L}=\mathscr{L}_{0} \oplus \mathscr{L}_{1}$ be a Leibniz superalgebra with nilindex $n+m$. Let $\left\{x_{1}, x_{2}, \ldots, x_{n}\right\}$ and $\left\{y_{1}, y_{2}, \ldots, y_{m}\right\}$ be a basis of $\mathscr{L}_{0}$ and $\mathscr{L}_{1}$ defined in (5). Suppose that the two generators belong in $\mathscr{L}_{1}$. Without loss of generality, we can assume that these generators are $\left\{y_{1}, y_{m_{1}+1}\right\}$.

Consider the following cases:

Case 1. Let $\left[y_{1}, y_{1}\right] \in \mathscr{L}_{0} \backslash \mathscr{L}_{0}^{2}$ be.

Then consider the Leibniz superalgebra $\left\langle y_{1}\right\rangle$ generated by the vector $y_{1}$. Since $\left[y_{1}, y_{1}\right] \in \mathscr{L}_{0} \backslash \mathscr{L}_{0}^{2}$, we can assume $\left[y_{1}, y_{1}\right]=x_{1}$. Then from (5), we deduce $\left\{x_{1}, x_{2}, \ldots, x_{n}, y_{2}, y_{3}, \ldots, y_{m_{1}}\right\} \subseteq\left\langle y_{1}\right\rangle$. It is easy to see that $y_{m_{1}+1} \notin\left\langle y_{1}\right\rangle$. Indeed, if $y_{m_{1}+1} \in\left\langle y_{1}\right\rangle$, then $\left\{y_{m_{1}+2}, \ldots, y_{m_{1}+m_{2}}\right\} \subseteq\left\langle y_{1}\right\rangle$ which implies $C(\mathscr{L}) \geq\left(n \mid m_{1}+\right.$ $\left.m_{2}, m_{3}, \ldots, m_{k}\right)$. That is a contradiction with the condition of characteristic sequence of $\mathscr{L}$, because $C(\mathscr{L})=\left(n \mid m_{1}, m_{2}, \ldots, m_{k}\right)$. Thus, the Leibniz superalgebra $\left\langle y_{1}\right\rangle$ is the vector space generated by $\left\{x_{1}, x_{2}, \ldots, x_{n}, y_{1}, y_{2}, \ldots, y_{m_{1}}\right\}$.

Since the superalgebra $\left\langle y_{1}\right\rangle$ is single-generated, then from Theorem 2.6, we have that either $m_{1}=n$ or $m_{1}=n+1$, and the multiplication in $\left\langle y_{1}\right\rangle$ has the following form:

$$
\begin{array}{ll}
{\left[x_{i}, x_{1}\right]=x_{i+1},} & 1 \leq i \leq n-1, \\
{\left[y_{j}, x_{1}\right]=y_{j+1},} & 1 \leq j \leq m_{1}-1, \\
{\left[x_{i}, y_{1}\right]=\frac{1}{2} y_{i+1},} & 1 \leq i \leq m_{1}-1, \\
{\left[y_{j}, y_{1}\right]=x_{j},} & 1 \leq j \leq n .
\end{array}
$$


Case $m_{1}=n$. Since $y_{1}$ and $y_{n+1}$ are the generators, we have

$$
\begin{aligned}
\mathscr{L} & =\left\{x_{1}, x_{2}, \ldots, x_{n}, y_{1}, \ldots, y_{n}, y_{n+1}, \ldots, y_{m}\right\}, \\
\mathscr{L}^{2} & =\left\{x_{1}, x_{2}, \ldots, x_{n}, y_{2}, \ldots, y_{n}, y_{n+2}, \ldots, y_{m}\right\} .
\end{aligned}
$$

The vector $x_{1}$ is not in $\mathscr{L}^{3}$. In fact, if $x_{1} \in \mathscr{L}^{3}$, then there exists $z \in \mathscr{L}_{1}$ such that $z \in \mathscr{L}^{2} \backslash \mathscr{L}^{3}$. Thereby $z \in\left\{\left[y_{1}, y_{1}\right],\left[y_{1}, y_{n+1}\right],\left[y_{n+1}, y_{1}\right],\left[y_{n+1}, y_{n+1}\right]\right\}$ and taking into account that $\left[y_{i}, y_{j}\right] \in \mathscr{L}_{0}$, we obtain $z \in \mathscr{L}_{0}$, which is a contradiction. Thus, $\mathscr{L}^{3}=\left\{x_{2}, \ldots, x_{n}, y_{2}, \ldots, y_{n}, y_{n+2}, \ldots, y_{m}\right\}$.

If $\mathscr{L}^{2 k}=\left\{x_{i}, x_{i+1}, \ldots, x_{n}, y_{j}, \ldots, y_{n}, y_{n+2}, \ldots, y_{m}\right\}$, then one can prove that $\mathscr{L}^{2 k+1}=\left\{x_{i+1}, \ldots, x_{n}, y_{j}, \ldots, y_{n}, y_{n+2}, \ldots, y_{m}\right\}$. In fact, if $z \in \mathscr{L}^{2 k} \backslash \mathscr{L}^{2 k+1}$, then $z$ has to be generated by $2 k$ products of the generators (but they are from $\mathscr{L}_{1}$ ). Hence this products belong to $\mathscr{L}_{0}$, and we have $z \in \mathscr{L}_{0}$.

Applying a similar argument, we get $\mathscr{L}^{2 k+2}=\left\{x_{i+1}, \ldots, x_{n}, y_{j+1}, \ldots, y_{n}\right.$, $\left.y_{n+2}, \ldots, y_{m}\right\}$.

Finally, we obtain $\mathscr{L}^{2 n+1}=\left\{y_{i_{1}}, y_{i_{2}}, \ldots, y_{i_{k}}\right\}$ and $\mathscr{L}^{2 n+2}=0$.

Since $\operatorname{dim}\left(\mathscr{L}^{2 n+1} \backslash \mathscr{L}^{2 n+2}\right)=1$, then $\mathscr{L}^{2 n+1}=\left\{y_{n+2}\right\}$, and the nilindex should be equal to $2 n+2$. Thus, $m=n+2$, and we have

$$
\begin{aligned}
\mathscr{L} & =\left\{x_{1}, x_{2}, \ldots, x_{n}, y_{1}, \ldots, y_{n}, y_{n+1}, y_{n+2}\right\}, \\
\mathscr{L}^{2 k} & =\left\{x_{k}, \ldots, x_{n}, y_{k+1}, \ldots, y_{n}, y_{n+2}\right\}, \quad 1 \leq k \leq n-1, \\
\mathscr{L}^{2 k+1} & =\left\{x_{k+1}, \ldots, x_{n}, y_{k+1}, \ldots, y_{n}, y_{n+2}\right\}, \quad 1 \leq k \leq n-1, \\
\mathscr{L}^{2 n} & =\left\{x_{n}, y_{n+2}\right\}, \quad \mathscr{L}^{2 n+1}=\left\{y_{n+2}\right\}, \quad \mathscr{L}^{2 n+2}=\{0\} .
\end{aligned}
$$

Furthermore, $\quad \mathscr{L}^{2 n}=\left[\mathscr{L}^{2 n-1}, \mathscr{L}\right]=\left\{\left[x_{n}, y_{1}\right], \quad\left[x_{n}, y_{n+1}\right], \quad\left[y_{n}, y_{1}\right], \quad\left[y_{n}, y_{n+1}\right]\right.$, $\left.\left[y_{n+2}, y_{1}\right],\left[y_{n+2}, y_{n+1}\right]\right\}$. We have $\left[x_{n}, y_{1}\right]=0$; otherwise we get a contradiction with characteristic sequence. Thus, the element $y_{n+2}$ can only be obtained from the product $\left[x_{n}, y_{n+1}\right]$. However,

$$
\left[x_{n}, y_{n+1}\right]=\left[\left[x_{n-1}, x_{1}\right], y_{n+1}\right]=\left[x_{n-1},\left[x_{1}, y_{n+1}\right]\right]+\left[\left[x_{n-1}, y_{n+1}\right], x_{1}\right]=0,
$$

and hence $\mathscr{L}^{2 n}=\left\{x_{n}\right\}$, which is a contradiction with the nilindex.

Case $m_{1}=n+1$. In this case, we also get a contradiction.

Case 2. Let $\left[y_{1}, y_{1}\right] \notin \mathscr{L}_{0} \backslash \mathscr{L}_{0}^{2}$ and $\left[y_{m_{1}+1}, y_{m_{1}+1}\right] \in \mathscr{L}_{0} \backslash \mathscr{L}_{0}^{2}$ be.

Then applying the same arguments for $y_{m_{1}+1}$ as for $y_{1}$ in Case 1, this contradicts the fact that both generators lie in $\mathscr{L}_{1}$, as well.

Case 3. Let $\left[y_{1}, y_{1}\right] \notin \mathscr{L}_{0} \backslash \mathscr{L}_{0}^{2}$ and $\left[y_{m_{1}+1}, y_{m_{1}+1}\right] \notin \mathscr{L}_{0} \backslash \mathscr{L}_{0}^{2}$ be.

Then, without loss of generality, we can assume that

$$
\begin{aligned}
& {\left[y_{1}, y_{m_{1}+1}\right]=x_{1},} \\
& {\left[y_{m_{1}+1}, y_{1}\right]=\sum_{i=1}^{n} b_{i} x_{i} .}
\end{aligned}
$$

If $b_{1}=1$, then making the change of basis $y_{1}^{\prime}=y_{1}+y_{m_{1}+1}$, we obtain $\left[y_{1}^{\prime}, y_{1}^{\prime}\right] \in$ $\mathscr{L}_{0} \backslash \mathscr{L}_{0}^{2}$. Therefore, this case can be reduced to Case 1 . 
If $\quad b_{1} \neq 1$, then $\left[y_{1}, y_{m_{1}+1}\right]-\left[y_{m_{1}+1}, y_{1}\right]=\left(1-b_{1}\right) x_{1}+b_{2} x_{2}+\cdots+b_{n} x_{n} \in$ $\mathscr{R}(\mathscr{L})$ and since $x_{i} \in \mathscr{R}(\mathscr{L}) \quad(2 \leq i \leq n)$ we get $x_{1} \in \mathscr{R}(\mathscr{L})$. From the Leibniz superidentity, we obtain $\mathscr{L}^{3}=\{0\}$ and, therefore, $n=1, m=2$.

According to Theorem 4.1, one generator lies in $\mathscr{L}_{0}$, and another one belongs to $\mathscr{L}_{1}$. Evidently, $x_{1}$ is a generator, and we can choose $y_{1}$ as the other generator.

Put

$$
\begin{array}{ll}
{\left[x_{i}, y_{1}\right]=\sum_{t=2}^{m} \alpha_{i, t} y_{t},} & 1 \leq i \leq n, \\
{\left[y_{j}, y_{1}\right]=\sum_{s=2}^{n} \beta_{j, s} x_{s},} & 1 \leq j \leq m .
\end{array}
$$

The following equality can be proved by induction:

$$
\left[y_{i}, y_{j}\right]=\sum_{s=0}^{\min \left\{i+j-1, m_{1}\right\}-i}(-1)^{s} C_{j-1}^{s} \sum_{t=2}^{n-j+s+1} \beta_{i+s, t} x_{t+j-s-1},
$$

where $1 \leq i, j \leq m_{1}$.

Theorem 4.2. Let $\mathscr{L}$ be a Leibniz superalgebra with characteristic sequence equal to $\left(n \mid m_{1}, m_{2}, \ldots, m_{k}\right)$, where $m_{1} \leq m-2$. Then the nilindex of $\mathscr{L}$ is less than $n+m$.

Proof. Let us suppose the opposite, i.e., the nilindex of $\mathscr{L}$ is $n+m$. Then $\operatorname{dim}\left(\mathscr{L}^{k}\right)=n+m-k$ with $2 \leq k \leq n+m$. In the adapted basis $\left\{x_{1}, x_{2}, \ldots, x_{n}, y_{1}, y_{2}, \ldots, y_{m}\right\}$, we have the following products

$$
\begin{aligned}
& {\left[x_{i}, x_{1}\right]=x_{i+1}, \quad 1 \leq i \leq n-1,} \\
& {\left[y_{j}, x_{1}\right]=y_{j+1}, \quad j \notin\left\{m_{1}, m_{1}+m_{2}, \ldots, m_{1}+m_{2}+\cdots+m_{k}\right\},} \\
& {\left[y_{j}, y_{1}\right]=\beta_{j, 2} x_{2}+\cdots+\beta_{j, n} x_{n}, \quad 1 \leq j \leq m,} \\
& {\left[x_{i}, y_{1}\right]=\alpha_{i, 2} y_{2}+\cdots+\alpha_{i, m} y_{m}, \quad 1 \leq i \leq n .}
\end{aligned}
$$

Suppose that $x_{2} \in \mathscr{L}^{3}$, i.e. $\mathscr{L}^{3}=\left\{x_{2}, \ldots, x_{n}, y_{3}, \ldots, y_{m}\right\}$. Then $x_{2}$ is generated from the products $\left[y_{j}, y_{1}\right]$, i.e., there exists $j_{0}\left(2 \leq j_{0} \leq m\right)$ such that $\beta_{j_{0}, 2} \neq 0$ in the product $\left[y_{j_{0}}, y_{1}\right]=\sum_{i=2}^{n} \beta_{j_{0}, i} x_{i}$.

Taking the change of basis $x_{1}^{\prime}=\frac{1}{\beta_{j_{0}, 2}}\left(\sum_{s=2}^{n} \beta_{j_{0}, s} x_{s-1}\right)$, we can assume that $\left[y_{j_{0}}, y_{1}\right]=x_{2}$.

The equalities

$$
\left[y_{j_{0}},\left[y_{1}, y_{1}\right]\right]=2\left[\left[y_{j_{0}}, y_{1}\right], y_{1}\right]=2\left[x_{2}, y_{1}\right] .
$$

and

$$
\left[y_{j_{0}},\left[y_{1}, y_{1}\right]\right]=\left[y_{j_{0}}, \sum_{i=2}^{n} \beta_{1, i} x_{i}\right]=0
$$

imply $\left[x_{2}, y_{1}\right]=0$. 
Since $\mathscr{L}^{3}=n+m-3$, we have $\mathscr{L}^{3}=\left\{x_{2}, x_{3}, \ldots, x_{n}, y_{3}, \ldots, y_{m_{1}}, A_{1,1} y_{2}+\right.$ $A_{1,2} y_{m_{1}+1}+\cdots+A_{1, k} y_{m_{1}+m_{2}+\cdots+m_{k-1}+1}, y_{m_{1}+2}, \ldots, y_{m_{1}+m_{2}}, A_{2,1} y_{2}+A_{2,2} y_{m_{1}+1}+\cdots+A_{2, k}$ $y_{m_{1}+m_{2}+\cdots+m_{k-1}+1}, \ldots, y_{m_{1}+m_{2}+\cdots+m_{k-1}}, \ldots, A_{k-1,1} y_{2}+A_{k-1,2} y_{3}+\cdots+A_{k-1, k} y_{m_{1}+m_{2}+\cdots+m_{k-1}+1}$, $\left.\ldots, y_{m}\right\}$.

If there exists $i$ such that

$$
\left[x_{i}, y_{1}\right] \equiv c_{2}\left(A_{i, 1} y_{2}+A_{i, 2} y_{m_{1}+1}+\cdots+A_{i, k} y_{m_{1}+\cdots+m_{k-1}+1}\right)\left(\bmod V_{3}\right)
$$

with $c_{2} \neq 0$, then applying the Leibniz superidentity for the elements $\left\{x_{i}, x_{1}, y_{1}\right\}$ inductively, we obtain $A_{i, 1}=0$ for all $i(1 \leq i \leq k-1)$.

Thus,

$$
\mathscr{L}^{3}=\left\{x_{2}, x_{3}, \ldots, x_{n}, y_{3}, \ldots, y_{m-1}, y_{m}\right\} .
$$

Consider the product $\left[x_{1}, y_{1}\right]=\sum_{i=2}^{n} \alpha_{1, i} y_{i}$. that

Case 1. Let $\alpha_{1,2} \neq 0$ be. Let us suppose that there exists $s$ for $3 \leq s \leq m_{1}$ such

$$
\begin{aligned}
\mathscr{L}^{s} & =\left\{x_{2}, \ldots, x_{n}, y_{s}, \ldots, y_{m}\right\}, \\
\mathscr{L}^{s+1} & =\left\{x_{3}, \ldots, x_{n}, y_{s}, \ldots, y_{m}\right\} .
\end{aligned}
$$

Note that $\beta_{s-1,2} \neq 0$, because $x_{2} \in \mathscr{L}^{s} \backslash \mathscr{L}^{s+1}$. The equality $\left[x_{2}, y_{1}\right]=0$ implies that the element $y_{s}$ must be generated by the products $\left[x_{i}, y_{1}\right]$ for $3 \leq i \leq n$. Therefore,

$$
\mathscr{L}^{s+2}=\left\{x_{4}, \ldots, x_{n}, y_{s}, \ldots, y_{m-1}, y_{m}\right\} .
$$

The parameters $\beta_{s, 2}, \beta_{s, 3}$ are equal to zero because $y_{s} \in \mathscr{L}^{s+2}$. Thus, we have

$$
\left[y_{s-1}, y_{1}\right]=\sum_{i=2}^{n} \beta_{s-1, i} x_{i} \quad \text { and } \quad\left[y_{s}, y_{1}\right]=\sum_{i=4}^{n} \beta_{s, i} x_{i} .
$$

From Eq. (6), we get

$$
\left[y_{2}, y_{s-1}\right] \equiv(-1)^{s-1} \beta_{s-1,2} x_{3}\left(\bmod U_{4}\right) .
$$

The following chain of the equalities

$$
\begin{aligned}
0 & =\left[x_{1},\left[y_{1}, y_{s-1}\right]\right]=\left[\left[x_{1}, y_{1}\right], y_{s-1}\right]+\left[\left[x_{1}, y_{s-1}\right], y_{1}\right] \\
& =\left[\sum_{i=2}^{n} \alpha_{1, i} y_{i}, y_{s-1}\right]+\left[\sum_{i=s}^{n} \gamma_{1, i} y_{i}, y_{1}\right] \equiv \alpha_{1,2} \beta_{s-1,2} x_{3}\left(\bmod U_{4}\right)
\end{aligned}
$$

implies $\alpha_{1,2} \beta_{s-1,2}=0$, which contradicts with the assumption $s \leq m_{1}$.

Thus, we have $s>m_{1}$. Then

$$
\begin{aligned}
\mathscr{L}^{m_{1}} & =\left\{x_{2}, \ldots, x_{n}, y_{m_{1}}, \ldots, y_{m}\right\}, \\
\mathscr{L}^{m_{1}+1} & =\left\{x_{2}, \ldots, x_{n}, y_{m_{1}+1}, \ldots, y_{m}\right\} .
\end{aligned}
$$


Since $\mathscr{L}^{m_{1}+2}=\left[\mathscr{L}^{m_{1}+1}, \mathscr{L}\right]$, then using Eq. (6), we conclude that

$$
\mathscr{L}^{m_{1}+2}=\left\{x_{3}, \ldots, x_{n}, y_{m_{1}+1}, \ldots, y_{m}\right\} .
$$

Therefore, $s=m_{1}+1$ and $\left[y_{m_{1}}, y_{1}\right]=\sum_{i=2}^{n} \beta_{m_{1}, 2} x_{2}$ with $\beta_{m_{1}, 2} \neq 0$. From (6), we get $\left[y_{2}, y_{m_{1}}\right] \equiv \beta_{m_{1}, 2} x_{3}\left(\bmod U_{4}\right)$ and using the Leibniz superidentity, we obtain $\beta_{m_{1}, 2}=0$, but it is a contradiction. Hence this case is not possible.

Case 2. Let $\alpha_{1,2}=0$ be. Then we have

$$
\begin{aligned}
{\left[x_{1}, y_{1}\right] } & =\alpha_{1,3} y_{3}+\cdots+\alpha_{1, m} y_{m}, \\
{\left[y_{1}, x_{1}\right] } & =y_{2}, \\
{\left[y_{1}, x_{1}\right]+\left[x_{1}, y_{1}\right] } & =y_{2}+\alpha_{1,3} y_{3}+\cdots+\alpha_{1, m} y_{m} \in \mathscr{R}(\mathscr{L}) .
\end{aligned}
$$

As in the previous case, we obtain $y_{i} \in \mathscr{R}(\mathscr{L})(2 \leq i \leq m)$. Applying the Leibniz superidentity for the elements $\left\{y_{j-1}, x_{1}, y_{1}\right\}$, we have

$$
\left[y_{j}, y_{1}\right]=\sum_{i=2}^{n+1-j} \beta_{j, i} x_{i+j-1}, \quad 2 \leq j \leq m_{1},
$$

which implies $\beta_{i, 2}=0$ for all $i$ with $2 \leq i \leq m_{1}$.

Since $y_{m_{1}+\cdots+m_{s-1}+1}(2 \leq s \leq k)$ is generated from the products $\left[x_{t}, y_{1}\right]$ with $1 \leq$ $t \leq n$, then we get $\beta_{i, 2}=0$ for $2 \leq i \leq m$, from the equality

$$
\left[\left[x_{t}, y_{1}\right], y_{1}\right]=\frac{1}{2}\left[x_{t},\left[y_{1}, y_{1}\right]\right]=\left[x_{t}, \sum_{i=2}^{n} \beta_{1, i} x_{i}\right]=0,
$$

and applying the Leibniz superidentity for the elements $\left\{y_{j-1}, x_{1}, y_{1}\right\}$. It contradicts assumption $x_{2} \in \mathscr{L}^{3}$.

Thus, we have $x_{2} \notin \mathscr{L}^{3}$, i.e.,

$$
\mathscr{L}^{3}=\left\{x_{3}, x_{4}, \ldots, x_{n}, y_{2}, y_{3}, \ldots, y_{m}\right\} .
$$
such that

Since $\left\{y_{2}, y_{m_{1}}, y_{m_{1}+m_{2}}, \ldots, y_{m_{1}+m_{2}+\cdots+m_{k}}\right\} \in \mathscr{L}^{3}$, then there exist $i_{1}, i_{2}, \ldots, i_{k} \geq 2$,

$$
\left|\begin{array}{lllll}
\alpha_{i_{1}, 2} & \alpha_{i_{1}, m_{1}+1} & \alpha_{i_{1}, m_{1}+m_{2}+1} & \cdots & \alpha_{i_{1}, m_{1}+m_{2}+\cdots+m_{k-1}+1} \\
\alpha_{i_{2}, 2} & \alpha_{i_{2}, m_{1}+1} & \alpha_{i_{2}, m_{1}+m_{2}+1} & \cdots & \alpha_{i_{2}, m_{1}+m_{2}+\cdots+m_{k-1}+1} \\
\vdots & \vdots & \vdots & & \vdots \\
\alpha_{i_{k}, 2} & \alpha_{i_{k}, m_{1}+1} & \alpha_{i_{k}, m_{1}+m_{2}+1} & \cdots & \alpha_{i_{k}, m_{1}+m_{2}+\cdots+m_{k-1}+1}
\end{array}\right| \neq 0 .
$$

Without loss of generality, we can assume that $\alpha_{i_{1}, 2} \neq 0$. Consider

$$
\left[x_{i_{1}}, y_{1}\right]+\left[y_{1}, x_{i_{1}}\right]=\sum_{t=2}^{m} \alpha_{i_{1}, t} y_{t} \in \mathscr{R}(L) .
$$


We obtain $y_{i} \in \mathscr{R}(\mathscr{L})$ with $2 \leq i \leq m$ multiplying by $x_{1}$ conveniently from the right side and taking into account condition (7).

Furthermore, proceeding with the computation of the brackets

$$
\begin{aligned}
& {\left[x_{2}, y_{1}\right]=} {\left[\left[x_{1}, y_{1}\right], x_{1}\right]=\alpha_{1,2} y_{3}+\cdots+\alpha_{1, m-1} y_{m}, } \\
& {\left[x_{3}, y_{1}\right]=} {\left[\left[x_{2}, y_{1}\right], x_{1}\right]=\alpha_{1,2} y_{4}+\cdots+\alpha_{1, m-2} y_{m}, } \\
& \vdots \\
& {\left[x_{n}, y_{1}\right]=\left[\left[x_{n-1}, y_{1}\right], x_{1}\right]=\alpha_{1,2} y_{n+1}+\cdots+\alpha_{1, n-m+1} y_{m}, }
\end{aligned}
$$

we obtain $y_{2} \notin \mathscr{L}^{3}$.

Thus, the superalgebra $\mathscr{L}$ with characteristic sequence $C(\mathscr{L})=$ $\left(n \mid m_{1}, m_{2}, \ldots, m_{k}\right)$ and $m_{1} \leq m-2$ has a nilindex less than $n+m$.

Combining the assertion of Theorem 3.2 and the classifications in [5, 8], we complete the classification of Leibniz superalgebras where the even part is a zero-filiform Leibniz algebra and with nilindex $n+m$. The obtained result allows us to consider the classification of Leibniz superalgebras of nilindex $n+m$ as an attainable problem.

\section{ACKNOWLEDGMENT}

Research partially supported by the PAICYT, FQM143 of Junta de Andalucía (Spain). The third author was supported by the grant NATO-Reintegration ref. CBP.EAP.RIG.983169.

\section{REFERENCES}

[1] Albeverio, S., Ayupov, Sh. A., Omirov, B. A. (2005). On nilpotent and simple Leibniz algebras. Comm. Algebra 33:159-172.

[2] Ancochea Bermudes, J. M., Gómez, J. R., Valeiras, G., Goze, M. (1996). Sur les composantes irréductibles de la variété des lois d'algèbres de Lie nilpotentes. (French) [Irreducible components of the variety of nilpotent Lie algebra laws] J. Pure Appl. Algebra 106:11-22.

[3] Ayupov, Sh. A., Omirov, B. A. (2001). On some classes of nilpotent Leibniz algebras. Siberian Math. J. 42:18-29.

[4] Ayupov, Sh. A., Khudoyberdiyev, A. Kh., Omirov, B. A. (2009). The classification of filiform Leibniz superalgebras of nilindex $n+m$. Acta Math. Sinica (English Series) 25:171-190.

[5] Camacho, L. M., Gómez, J. R., Navarro, R. M., Omirov, B. A. (2010). Classification of some nilpotent class of Leibniz superalgebras. Acta Math. Sinica (English Series) 26:182201.

[6] Cuvier, C. (1994). Algèbres de Leibnitz: définitions, propriétés. Ann. Sci. École Norm. Sup. (4) $27: 1-45$.

[7] Dzhumadil'daev, A. S. (1999). Cohomologies of colour Leibniz algebras: Pre-simplicial approach, Lie Theory and Its Applications in Physics III. Proceedings of the Third International Workshop, pp. 124-135.

[8] Gómez, J. R., Navarro, R. M., Omirov, B. A. On nilpotent Leibniz Superalgebras. arXiv:math/0611723. 
[9] Gómez, J. R., Khakimjanov, Yu., Navarro, R. M. (2004). Some problems concerning to nilpotent Lie superalgebras. J. Geom. Phys. 51:473-486.

[10] Goze, M., Ancochea Bermudez, J. M. (2001). On the classification of rigid Lie algebras. J. Algebra 245:68-91.

[11] Goze, M., Goze, N., Remm, E. (2010). n-Lie algebras. African Journal of Mathematical Physics 8:178-28.

[12] Goze, M., Khakimdjanov, Yu. (1996). Nilpotent Lie Algebras. Mathematics and its Applications, Vol. 361. Dodrecht: Kluwer Academic Publishers Group.

[13] Humphreys, J. E. (1975). Linear Algebraic Groups. Graduate Texts in Math., Vol. 21, New York-Heidelberg: Springer-Verlag, p. 247.

[14] Kac, V. G. (1977). Lie superalgebras. Advances in Math. 26:8-96.

[15] Loday, J.-L. (1993). Une version non commutative des algèbres de Lie: les algèbres de Leibniz. Ens. Math. 39:269-293.

[16] Sagle, A. A. (1961). Malcev algebras. Trans. Amer. Math. Soc. 101:426-458. 